Methods During 2013-2015, 1278 adults seeking accommodation in East Village were recruited (participation rate 70\%, $\mathrm{n}=1278 / 1819)$; 520 were seeking social housing, 524 intermediate (affordable) and 234 market-rent accommodation. Participants were followed-up after 2 years; those who moved into East Village formed the intervention group, and those who did not move to East Village controls. Self-reported mental-health (depression, anxiety), subjective well-being (life satisfaction, worthwhile, happiness), and neighbourhood perceptions (quality and crime-free) were assessed by questionnaire. Multilevel linear regression models examined change in these outcomes adjusted for age, sex, ethnicity and household (random effect), comparing those in the intervention group with controls, overall and by housing sector.

Results Of 1278 adults recruited at baseline, 877 (69\%) adults were followed-up after two years; half $(n=440 / 877)$ had moved to East Village. There were marginally lower levels of depression and anxiety amongst those who moved to East Village, compared with those who did not, but differences were not statistically significant. Overall levels of positive well-being (including life satisfaction, worthwhile, happiness) were marginally higher amongst participants who moved to East Village, but again differences were not statistically significant. However, increases in life satisfaction and happiness amongst those living in intermediate accommodation in East Village were stronger $(p=0.01, p=0.05$ respectively $)$. The most marked differences were in neighbourhood perceptions, where sizeable increases in both quality and crime-free status were observed amongst those living in East Village compared with those who were not, overall and in each housing sector (all $\mathrm{P}$ values <0.01). There was also the suggestion that improvements in crime-free perceptions were stronger in the social and intermediate sectors compared to those in market-rent accommodation (test for interaction, $\mathrm{p}=0.04$ ).

Conclusion East Village had modest effects on measures of well-being, but appreciable effects on neighbourhood perceptions. Longer-term exposure to better neighbourhoods could plausibly have beneficial effects for health, particularly for both mental health and well-being, more so among those from less privileged circumstances, who potentially have the most to gain.

\section{RF10 CHRONIC INFLAMMATION AND SUBSEQUENT DEPRESSIVE SYMPTOMS: THE MEDIATING ROLE OF SLEEP}

P Frank*, A Kaushal, D Cadar. Department of Behavioural Science and Health, University College London, UK

\subsection{6/jech-2018-SSMabstracts.99}

Background Systemic inflammation has been associated with the onset of depressive symptoms. However, the exact mechanisms underlying the relationship between inflammation and depression remain elusive. This study examined whether sleep explained the association between elevated levels of inflammatory markers and subsequent depressive symptoms in an English nationally representative sample.

Methods The sample consisted of 2953 men and women (aged 50+) recruited from the English Longitudinal Study of Ageing (ELSA) an ongoing, open, representative prospective cohort study. Four waves of data between 2008/09 (wave 4) and 2016/17 (wave 8) were analysed. Serum levels of inflammatory markers (C-reactive protein (CRP)) and covariates (age, sex, education, wealth, body mass index, smoking, cholesterol, triglyceride) were measured at wave 4 (considered here as the baseline). Self-reported sleep disturbance (vs no sleep disturbance) was examined via three items of the Jenkins Sleep Problems Scale (difficulty falling asleep, trouble remaining asleep and morning tiredness) at a four-year follow-up (wave 6, 2012/13). Depressive symptoms were assessed at baseline and six years later (wave 7, 2014/15) using the 8item version of the Centre for Epidemiological Studies Depression Scale (CES-D) (excl. the item on sleep). Binary mediation analysis was used to investigate whether sleep mediated the relationship between systemic inflammation and depressive symptoms, adjusting for the full set of covariates.

Results High baseline levels of CRP were significantly associated with greater odds of subsequent depressive symptoms, independent of age, sex and baseline depressive symptoms (Odds Ratio $(\mathrm{OR})=1.32 \quad(95 \%$ Confidence Intervals (CI)) 1.02-1.70). Further adjustment for socio-economic variables (education, wealth status) attenuated this relationship to nonsignificance $(\mathrm{OR}=1.17$ (95\% CI) 0.90 to 1.53$)$. Moreover, high CRP levels at baseline were significantly related to higher odds of reporting sleep disturbance at wave $6(\mathrm{OR}=1.44$ (95\% CI) 1.14 to 1.82 ). Sleep disturbance was associated with greater odds of subsequent depressive symptoms $(\mathrm{OR}=2.69$ (95\% CI) 2.03 to 3.57 ). Mediation analyses revealed that sleep problems mediated the relationship between high CRP and depressive symptoms, explaining a total of $65.61 \%$ of this association.

Conclusion Our results showed that sleep acted as a strong partial mediator of the relationship between elevated levels of CRP and subsequent depressive symptoms in an English nationally representative sample. Targeting sleep disturbance via tailored interventions may be effective in alleviating inflammation-associated depressive symptoms.

\section{RF11 THE ASSOCIATION BETWEEN DIFFERENT MEASURES OF DEPRESSION AND SUBSEQUENT MAJOR CARDIOVASCULAR EVENTS}

R Prigge*, S Wild, CA Jackson. Usher Institute of Population Health Sciences and Informatics, The University of Edinburgh, Edinburgh, UK

\subsection{6/jech-2018-SSMabstracts. 100}

Background Associations between depression and cardiovascular disease have been reported in a number of studies. However, in many of these, the results have not been adjusted for potentially important confounding factors. With the large amount of data collected, the UK Biobank offers a unique opportunity to investigate to what extent different measures of depression remain independent risk factors for major cardiovascular events (MCVE) after controlling for a number of potential confounding factors.

Methods We used data from 275,759 UK Biobank participants without a history of cardiovascular diseases and major mental disorders other than depression who had complete data available. In primary analyses, depression was defined as one or more of self-reported depression, antidepressant use, or hospital admission for depression. In secondary analyses, the effects of each of the subcategories of depression were analysed separately. MCVE were defined as first-ever fatal or non-fatal stroke or myocardial infarction ascertained from hospital 
admission and cause of death records. We performed Cox Proportional Hazards models to estimate the risk of MCVE among participants with depression compared to those without depression. Fully adjusted models included measures of age, sex, ethnicity, education, income, area-based deprivation, body mass index, alcohol intake, physical activity, smoking, homeownership, fruit and vegetable consumption, oily fish intake, and family history of stroke, heart disease, hypertension and/ or depression.

Results We identified a total of 21842 (7.9\%) participants with depression at baseline. During a median of 6.7 years of follow-up, an incident MCVE occurred among 326 participants with depression and 3718 participants without depression. In fully adjusted models, hazard ratios $(95 \%$ confidence intervals) for MCVE were 1.13 (1.01-1.27) for participants with any indication of depression, 1.14 (1.00-1.31) for participants with self-reported depression, 1.60 (1.18-2.17) for participants with history of a hospital admission with depression, and $1.12(0.99-1.27)$ for participants reporting antidepressant use. Similar patterns were observed when stroke and myocardial infarction were used separately as outcomes.

Conclusion All measures of depression remained independent risk factors for MCVE after adjustment for a variety of potential confounding factors and effect estimates were similar for all sub-categories of depression. The adjusted hazard ratios should only be interpreted causally if one assumes that the covariates are common sources of depression and MCVE. This assumption remains controversial. Future studies should apply more advanced statistical methods in order to determine the effect of lifestyle factors as potential mediators and explore potential for interactions.

\section{\begin{tabular}{|l|l}
\hline RF12 & ADULT HEIGHT AND RISK OF INCIDENT ATRIAL
\end{tabular} FIBRILLATION AND OF INCIDENT HEART FAILURE IN OLDER MEN: THE BRITISH REGIONAL HEART STUDY}

${ }^{1}$ SG Wannamethee*, ' $\mathrm{OA}$ Papacosta, ' $\mathrm{LT}$ Lennon, ${ }^{2} \mathrm{PH}$ Whincup. ${ }^{1}$ Primary Care and Population Health, UCL, London, UK; ${ }^{2}$ Population Health Research Institute, St George's University of London, London, UK

\subsection{6/jech-2018-SSMabstracts. 101}

Background Heart failure (HF) is one of the leading causes of mortality, morbidity and hospitalisation in older adults. Although short stature has been associated with increased risk of coronary heart disease previous studies have consistently shown tall stature to be associated with increased risk of atrial fibrillation (AF) a known major risk factor for the development of HF. Relatively few studies have investigated the association between height and incident HF. We have therefore examined prospectively the association between adult height and incident $\mathrm{AF}$ and incident $\mathrm{HF}$ in a population based cohort of older men.

Methods Prospective study of 3530 men aged 60-79 years with no diagnosed HF, myocardial infarction or stroke at baseline (1998-2000) followed up for a mean period of 15 years, in which there were 212 incident HF cases. Incident AF was based on a subgroup of men $(n=1348)$ who attended re-examination in 2010-2012. Men were divided into 5 height groups: <168.2, 168.2-172.9, 173.0-176.9, 177.0 183.0 and $>183.0 \mathrm{cms}$ based on the quartile distribution of height with the top 5 percent separated out.

Results CVD risk factors tended to decrease with increasing height but a positive association was seen between height and prevalent AF. Tall stature was prospectively associated with increased risk of incident AF. Both short stature $(<168.2 \mathrm{cms})$ and tall stature $(>183.0 \mathrm{cms})$ were associated with significantly increased risk of $\mathrm{HF}$ in age-adjusted analysis compared to those in the second height quartile [HR (95\% CI) 1.58 $(1.07,3.02)$ and $1.90(1.04,3.50)$ respectively]. The increased risk seen in short men was attenuated after adjustment for lifestyle characteristics, established CHD risk factors, inflammation (CRP) and prevalent AF [adjusted $\mathrm{HR}=1.37 \quad(0.92,2.02)$ ]. Since tall men had the most favourable CHD risk factors, adjustment increased the risk further (adjusted HR (95\% CI) 1.97 (1.05,3.68). However further adjustment for incident AF attenuated the increased risk seen in tall men $(\mathrm{HR}=1.76$ $(0.93,3.31)]$.

Conclusion Both short stature and tall stature are associated with increased $\mathrm{HF}$ risk but the pathways underlying these associations are different. The increased risk of HF in short adults appear to be largely explained by adverse CVD risk factors associated with short stature; in tall men the association was partially explained by their increased risk of developing AF. Average body height has increased worldwide over the decades and if this trend continues, the prevalence of tall older adults is likely to increase which may contribute to an increasing burden of $\mathrm{AF}$ and $\mathrm{HF}$.

\section{RF13 THE IMPACT OF LIFE EVENTS ON LATER LIFE: A LATENT CLASS ANALYSIS OF THE ENGLISH LONGITUDINAL STUDY OF AGEING}

00 Enwo*, J Ford, E Player, N Steel. Norwich Medical School, University of East Anglia, Norwich, UK

\subsection{6/jech-2018-SSMabstracts. 102}

Background Events over an individual's life course can positively or negatively affect their health and wellbeing in older age. We aimed to identify associations between groups of older people with similar life events and their health, behaviours and social interactions in older age.

Methods We undertook a latent class analysis of the English Longitudinal Study of Ageing wave 3. Groups of participants with similar life events were generated based on eight life events; maternal and paternal closeness, educational opportunities in childhood, financial hardship, bereavement due to war, involvement in conflict, violence, and experiencing a natural disaster. The number of groups was determined based on model fit and team discussion. Linear and logistic regression were used to explore associations between these groups and pre-specified health and wellbeing factors, adjusted for age, gender, ethnicity and socioeconomic class and weighted for group probability.

Results 7555 participants were allocated to one of four groups: Group A 'few life events' $(n=6,250)$, Group B 'emotionally cold mother' $(n=724)$, Group C 'violence in combat' $(n=274)$ and Group D 'many life events' $(n=307)$. Participants in Group D were statistically significantly more likely than those in Group A to experience disability (coefficient $0.35,95 \%$ CI 0.20 to 0.50 ), reduced quality of life (coefficient $-5.33,95 \%$ CI -6.61 to -4.05$)$, psychological disorders (OR 3.01, 95\% CI 2.18 to 4.17) and social detachment (OR 2.60, 95\% CI 1.36 to 4.97). Group C membership compared to Group A was associated with reduced quality of life (coefficient $-1.95,95 \% \mathrm{CI}-3.08$ to -0.82 ) and a life-threatening 